\title{
SISTEMA DE INFORMACIÓN DE LA RED NACIONAL DE LABORATORIOS DE SALUD PÚBLICA EN EL PERÚ (NETLAB)
}

\author{
Javier Vargas-Herrera',2,a, José Segovia-Juarez ${ }^{3, b}$, Gladys María Garro Nuñez $2, c^{2}$
}

\begin{abstract}
RESUMEN
Los sistemas de información de laboratorio clínico, producen mejoras en la calidad de la información, la reducción de los costos del servicio, disminución de la espera para obtener resultados, entre otros. En el proceso de construcción de este sistema de información, el Instituto Nacional de Salud (INS) del Perú ha desarrollado e implementado un aplicativo a través de Internet basado en la web, para comunicar, al personal de salud (laboratoristas, epidemiólogos, gestores de estrategias sanitarias, médicos tratantes, etc.), los resultados de las pruebas de laboratorio que se realizan en el INS o en los laboratorios de la Red Nacional de Laboratorios de Salud Pública el cual es llamado NETLAB. Este artículo presenta la experiencia de la implementación de NETLAB, su situación actual y las perspectivas de su empleo, así como su contribución en la prevención y control de enfermedades en el Perú.
\end{abstract}

Palabras clave: Sistemas de información de laboratorio; Informática médica; Prevención y control (fuente: DeCS BIREME).

\section{INFORMATION SYSTEM OF THE NATIONAL NETWORK OF PUBLIC HEALTH LABORATORIES IN PERU (NETLAB)}

\begin{abstract}
Clinical laboratory information systems produce improvements in the quality of information, reduce service costs, and diminish wait times for results, among other things. In the construction process of this information system, the National Institute of Health (NIH) of Peru has developed and implemented a web-based application to communicate to health personnel (laboratory workers, epidemiologists, health strategy managers, physicians, etc.) the results of laboratory tests performed at the Peruvian NIH or in the laboratories of the National Network of Public Health Laboratories which is called NETLAB. This article presents the experience of implementing NETLAB, its current situation, perspectives of its use, and its contribution to the prevention and control of diseases in Peru.
\end{abstract}

Key words: Clinical laboratory information systems; Medical informatics; Prevention and control (source: MeSH NLM).

\section{LOS SISTEMAS DE INFORMACIÓN DE LABORATORIO EN EL INSTITUTO NACIONAL DE SALUD}

El sistema de información de laboratorio, LIS del inglés Laboratory Information System, como se le denomina en el lenguaje de la salud electrónica (eSalud), corresponde a la sección de la historia clínica electrónica, que tiene la función de gestionar los datos del laboratorio que interopera con los datos clínicos (1). La aplicación de tecnologías de la información y comunicaciones (TIC) al proceso de producción de los sistemas de laboratorio, se viene implementando desde las últimas décadas del siglo $X X{ }^{(2,3,4)}$, lo cual ha producido mejoras en la calidad de la información, en la reducción de los costos del servicio, en la disminución de los tiempos de espera para obtener resultados, en el monitoreo del estado de salud de los pacientes, en la medición de la productividad del laboratorio, además que aportan efectivamente al proceso de toma de decisiones clínicas y de salud pública.

Dentro de la estructura de los establecimientos de salud, el laboratorio es un órgano de apoyo para el diagnóstico, por lo que su nivel de complejidad debe estar acorde con el del establecimiento al que pertenece, a los recursos humanos con los que cuenta y a los materiales y equipos disponibles. El Instituto Nacional de Salud (INS), es el rector del sistema de la red nacional de laboratorios

\footnotetext{
Facultad de Medicina. Universidad Nacional Mayor de San Marcos. Lima, Perú

Ministerio de Salud. Lima, Perú.

Oficina General de Información y Sistemas. Instituto Nacional de Salud. Lima, Perú.

Medico epidemiólogo; ${ }^{\mathrm{b}} \mathrm{PhD}$ en Ciencias de la Computación; ${ }^{\mathrm{c}}$ enfermera epidemióloga

Recibido: : 22-04-15 Aprobado: 13-05-15
}

Citar como: Vargas-Herrera J, Segovia-Juarez J, Garro Nuñez GM. Sistema de información de la red nacional de laboratorios de salud pública en el Perú (NETLAB). Rev Peru Med Exp Salud Publica. 2015;32(2):378-84. 


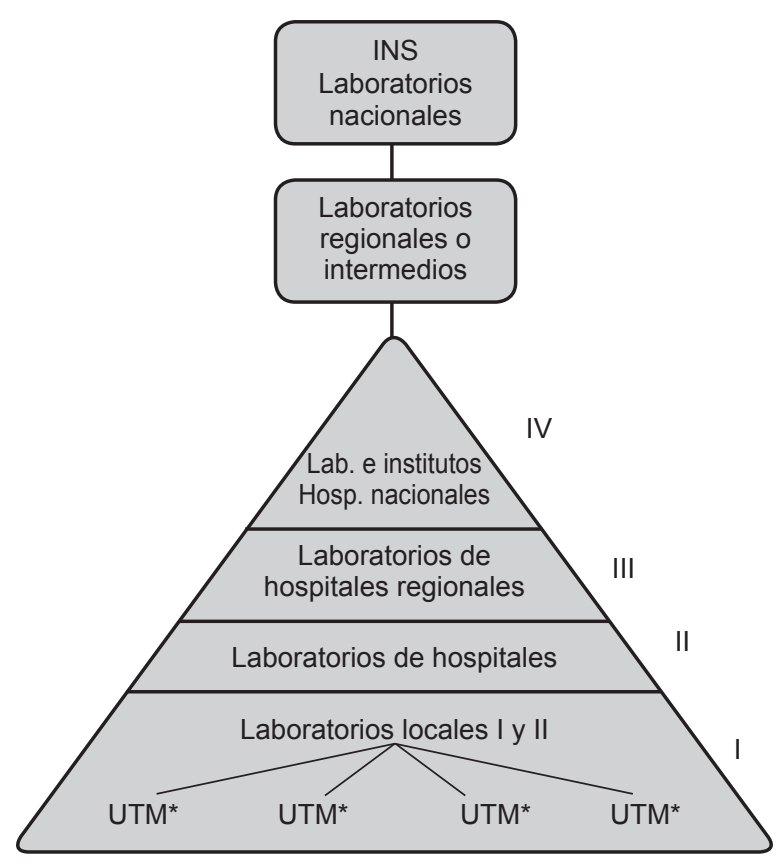

Figura 1. Organización de la Red Nacional de Laboratorios de Salud Pública en el Perú

* UTM: Unidades tomadoras de muestras.

de salud pública; de ahí surge la importancia de contar con un sistema de información único e interconectado que se ajuste a su organización. Por ello, es necesario conocer la estructura de la Red Nacional de Laboratorios de Salud Publica en el país (Figura 1).

EI INS ha transitado, desde mediados de la última década del siglo pasado, el proceso de construir su LIS (Figura 2). Inicialmente, en los años 1998-1999, se diseñó un aplicativo de red local que simplemente almacenaba los datos del laboratorio para su impresión y entrega a los usuarios denominado SISLAB, este aplicativo era solo de uso del INS y estaba desarrollado en FOX PRO 2.6 para DOS que luego migró a Visual FOX PRO 5.O, para Windows 95, pero que no tenía todas las funcionalidades de una base de datos relacional. Posteriormente, en el año 2000, con el apoyo del programa de vigilancia global de enfermedades emergentes del Departamento de Defensa de los Estados Unidos (DoD GEIS en inglés) se implementó un aplicativo informático que funcionaba en una red informática local, denominado PHLIS (Public Health Laboratory Information System) ${ }^{(5,6)}$ desarrollado por los Centros de Control de Enfermedades de Atlanta, (CDC).

El PHLIS era un aplicativo cliente-servidor que cumplía con los estándares de un LIS, es decir, con los requerimientos preanalíticos (solicitud, obtención de muestras), analíticos (procesamiento, registro de resultados) y posanalíticos (impresión y edición de reportes de resultados, archivo electrónico de los datos de las muestras y resultados de las pruebas). Sin embargo, los reportes que emitía eran impresos en papel y tenía limitaciones para el intercambio adecuado de información, a través de Internet, con los establecimientos solicitantes, en consecuencia, los resultados llegaban retrasados, afectaban la calidad del servicio y significaban un impacto negativo para la atención de los pacientes. Este aplicativo fue utilizado en el INS y en los laboratorios regionales de Ayacucho y San Martín.

Esta situación apresuró en el año 2006, el diseño y desarrollo de un aplicativo LIS basado en la web, al que se denominó NETLAB. Un factor determinante para su implementación fue la necesidad de entregar, en tiempo real, resultados de las pruebas de carga viral y recuento

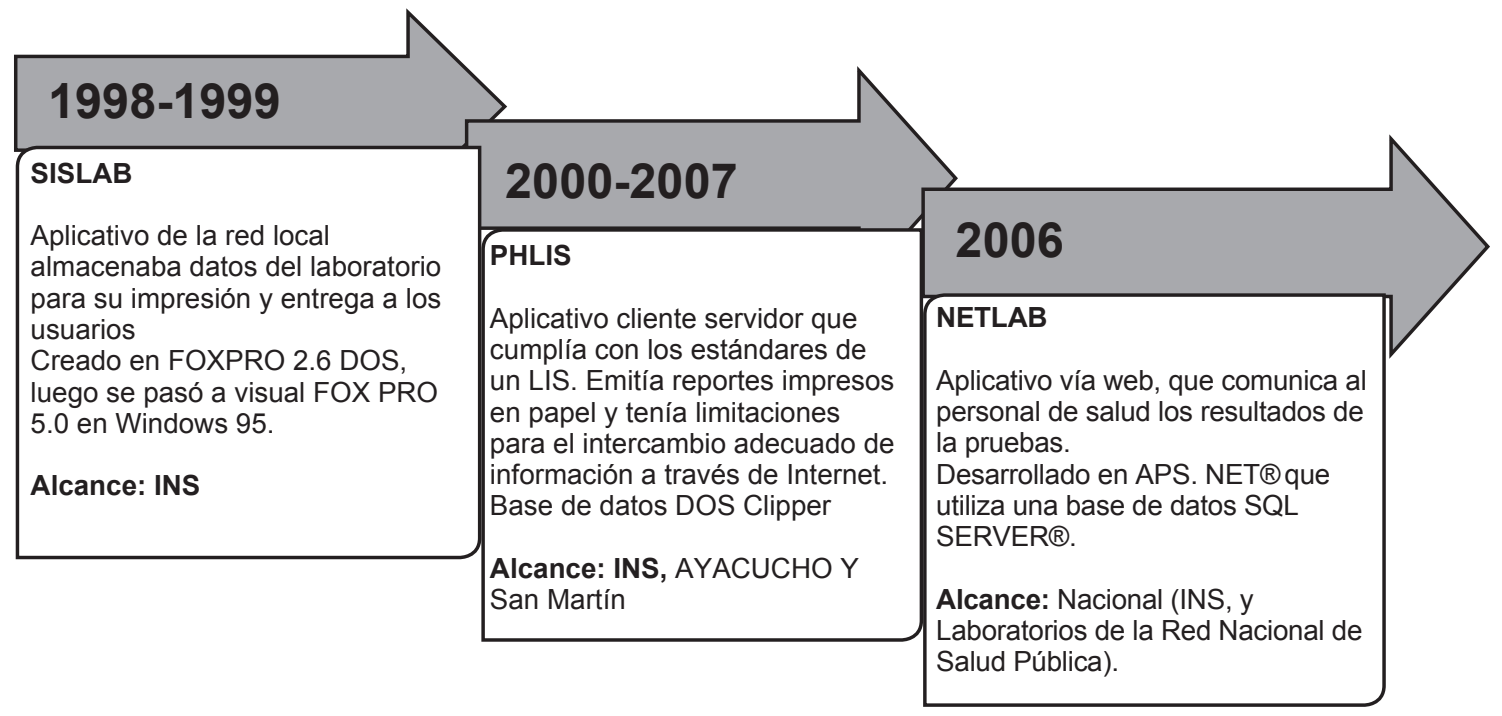

Figura 2. Evolución de los sistemas de información de laboratorio en el INS 
de linfocitos CD4, para el monitoreo de personas viviendo con $\mathrm{VIH}$ sida en tratamiento antirretroviral, que se había iniciado como programa gratuito a cargo del Estado ${ }^{(7,8)}$. Otro factor importante fue la exploración de la disponibilidad de Internet en los laboratorios regionales y establecimientos de salud urbanos, así como su uso entre los trabajadores ${ }^{(9)}$.

Con el desarrollo de este aplicativo, el INS fue pionero en el uso de las TIC en la historia de los sistemas de información en salud del Ministerio de Salud (MINSA), enfrentando los retos que plantea la eSalud (10,11). La principal funcionalidad de NETLAB consiste en comunicar, a través de Internet, al personal de salud (laboratoristas, epidemiólogos, gestores de estrategias sanitarias, médicos tratantes, etc.), resultados de las pruebas de laboratorio que se solicitan al INS o a laboratorios de la Red Nacional de Laboratorios de Salud Pública. Los resultados de las pruebas de laboratorio corresponden, principalmente, a la confirmación o el descarte de enfermedades bajo vigilancia epidemiológica o de importancia para la salud pública y, en otros casos, al monitoreo clínico del tratamiento de pacientes con enfermedades crónicas. El presente artículo tiene como objetivo, presentar la experiencia en la implementación de NETLAB, su situación actual y las perspectivas de su empleo en la Red Nacional de Laboratorios de Salud Pública, así como su contribución en la prevención y control de enfermedades en el Perú.

\section{EXPERIENCIA DEL PROCESO DE IMPLEMENTAR NETLAB}

En enero de 2007 se inició la operación de NETLAB, un aplicativo basado en la web desarrollado en ASP. $\mathrm{NET}^{\circledR}$ que utiliza una base de datos SQL Server ${ }^{\circledR}$, y que reemplazó al PHLIS como LIS del INS. En su diseño, se revisó el ciclo completo del procesamiento de las muestras y de la producción de resultados, y se identificaron los procesos que no aportaban valor agregado al flujo de trabajo, así como aquellos que demandaban tiempo excesivo. Se identificaron como nudos críticos: 1) Existencia de dos sistemas de registro; uno para las muestras clasificadas como "no conformes", debido a la detección de fallas en su embalaje, conservación o transporte, y el otro, para las muestras "conformes"; 2) Codificación manual de las muestras y de sus alícuotas; 3) Almacenamiento prolongado de la muestra en el área de recepción; 4) Tiempo excesivo entre la admisión de la muestra y el registro de resultados; 5) Falta de verificación de los resultados registrados; 6) Publicación de resultados en la web mediante procesos manuales, y 7) Deficiente proceso de impresión de resultados en papel. El desarrollo de NETLAB se enfocó en la resolución de esos nudos críticos, lo que favoreció que los laboratorios integrantes del Sistema de la Red Nacional de Laboratorios interactúen con el INS a través del sistema de información, facilitando la resolución de problemas relacionados a la referencia y contrarreferencia de las muestras.

Uno de los principales problemas que se presentó durante el proceso de implementación de NETLAB fue cómo reemplazar las firmas manuscritas en los reportes de resultados si se utilizan formatos digitales. Si bien en el Perú existía, desde el año 2000, la Ley de Firmas y Certificados Digitales, 27269, esta no había sido reglamentada, sin embargo, existían antecedentes del uso de firmas electrónicas en otras entidades del Estado como la Superintendencia Nacional de Aduanas y de Administración Tributaria (SUNAT) o la Contraloría General de la República, las cuales se utilizaban bajo un acuerdo entre las partes, es decir, entre la entidad y el administrado. La diferencia entre una firma electrónica y una firma digital, es que esta última cuenta con un certificado digital, expedido por una entidad certificadora acreditada, que garantiza la identidad del firmante y el no repudio de la firma. En el INS se optó por esta modalidad de firma electrónica, sin embargo, se implementó el certificado digital del sitio web de NETLAB. En la actualidad la Ley 27269 ya cuenta con su reglamento y en el INS se viene utilizando la firma digital para algunos procesos administrativos, por lo tanto, es un reto implementar la firma digital en los resultados NETLAB.

EI NETLAB tiene la capacidad de enviar mensajes de correo electrónico y de texto SMS. En el caso de los mensajes de correo electrónico, se puede programar enviarlos a los usuarios, incluidos los pacientes, para informarles sobre el estado de sus muestras o la disponibilidad de resultados para consultar el sistema, sin embargo, se requiere mantener actualizada la base de datos de los usuarios y el consentimiento de estos para recibirlos, aspectos que no se mantienen adecuadamente. En cuanto a los mensajes de texto SMS, se utilizaron durante la epidemia de influenza del año 2009, para informar los resultados de pruebas negativas a la infección, lo que mostró la potencialidad de esta herramienta, no obstante, no están definidas las políticas de su empleo ni se mantiene un presupuesto que permita financiar este servicio.

La experiencia NETLAB ha sido evaluada por "Ciudadanos al Día", un organismo no gubernamental, que con el apoyo de la Defensoría del Pueblo y la International Finance Corporation (IFC), organizan un concurso anual de las Buenas Prácticas en Gestión Pública (Premio BPG) en el Perú, premiándola con 
el primer puesto del concurso en el año 2008, en la categoría de "Servicios de Atención al Ciudadano" y quedando finalista en el año 2014, en el "Premio de la década", que se otorga a las buenas prácticas que se consolidan y mantienen en el tiempo ${ }^{(12)}$.

\section{EL NETLAB EN LA ACTUALIDAD}

El sistema de información NETLAB inició su producción el 30 de enero de 2007, registrando información solo de pruebas relacionadas con el diagnóstico o monitoreo de VIH Sida; progresivamente, se fueron incorporando todas las pruebas de laboratorio para el diagnóstico y monitoreo de enfermedades. Hasta el año 2014, se han registrado un total de 914315 muestras para la realización de pruebas de diagnóstico de 122 enfermedades de interés en salud pública, sin contar las muestras procesadas para el control de calidad, el monitoreo clínico de los pacientes crónicos o la evaluación del desempeño de los analistas.

La implantación del NETLAB implicó la optimización del proceso de admisión y la distribución de las muestras desde el área de recepción y obtención, hasta los laboratorios que ejecutan las pruebas. Asimismo, se implementó un sistema de código de barras para identificar, inicialmente, a las muestras que ingresaban a los laboratorios de referencia nacional, pero posteriormente, se incluyó también, a las muestras procedentes de los laboratorios regionales y locales donde se atienden los pacientes. En este código, se incorporó el código del Registro Nacional de Establecimientos de Salud (RENAES), para asegurar que las muestras enviadas al laboratorio sean identificadas por el establecimiento solicitante. Lo relevante de esta práctica es que permite la trazabilidad de una muestra a lo largo del proceso, relacionándola con las pruebas que se realicen en los laboratorios de referencia regional y nacional del INS.

Con el NETLAB el promedio del tiempo de respuesta de la publicación de resultados vía web disminuyó sustancialmente. Por ejemplo, un resultado de carga viral o recuento de linfocitos CD4 que antes de la implementación demoraba una mediana de 21 días, contados desde el recibo de la muestra por el INS hasta el registro del resultado en el sistema de información, y más de dos meses hasta su disponibilidad en formatos en papel; hoy está disponible en menos de un día (7). Otra ventaja de estar en web, es que permite aprovechar el uso de un servicio web para cargar los datos de identificación de los pacientes, al ingresar el número del Documento Nacional de Identidad (DNI), gracias a un convenio entre el MINSA y el Registro Nacional

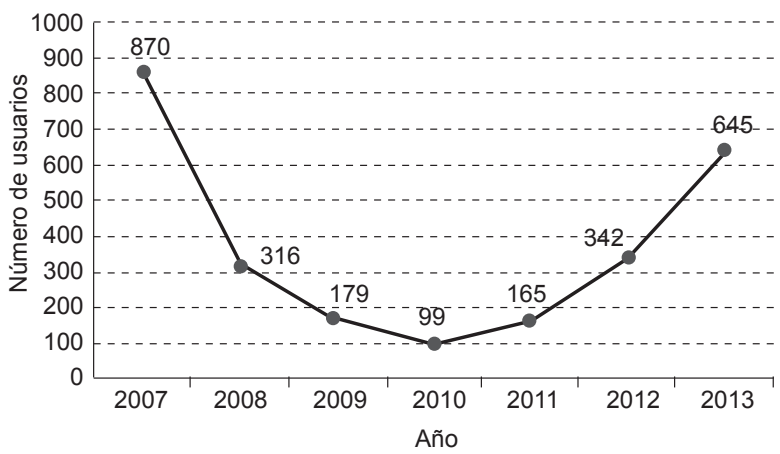

Figura 3. Número de usuarios activos de NETLAB, por año Fuente: Sistema de Información NETLAB

de Identificación y Estado Civil (RENIEC). Este tipo de consulta esta activa desde el año 2011.

La Figura 3, muestra la evolución en el número de usuarios, pacientes y profesionales de la salud que cuentan con clave de acceso al sistema NETLAB a diciembre de 2014. Inicialmente, en el año 2007, entre los usuarios del sistema se incluyó a los ciudadanos viviendo con VIH Sida (PVVS), quienes podían monitorear directamente y de manera gráfica la evolución de su tratamiento. Este aspecto generó una discusión sobre el derecho a la información sobre su propia salud que podrían tener los pacientes. Por ello, de manera progresiva, se dejó de promover este servicio entre los usuarios PVVS y se fue disminuyendo la cantidad de usuarios pacientes. Actualmente, los usuarios son principalmente personal de salud que opera en los servicios de atención, epidemiólogos y administradores de las estrategias sanitarias en los niveles regional y nacional

La Figura 4, muestra la evolución de las muestras estudiadas para el diagnóstico de enfermedades.

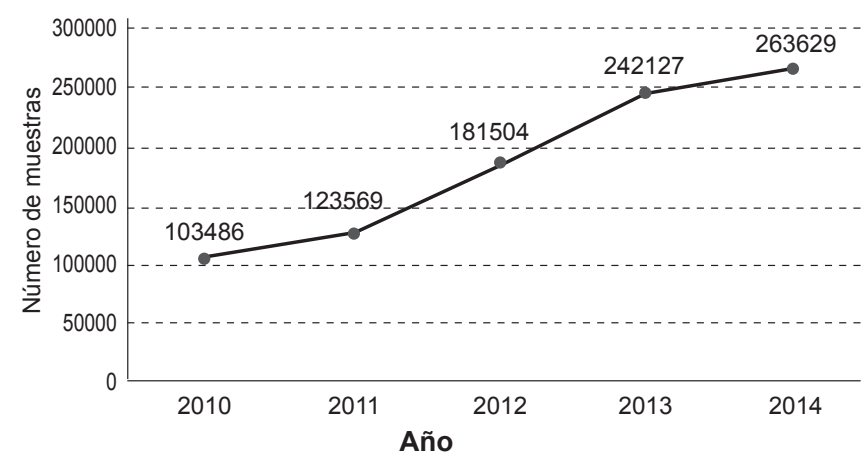

Figura 4. Número de muestras registradas en el NETLAB. Años 2010 al 2014

Fuente: Sistema de Información NETLAB 


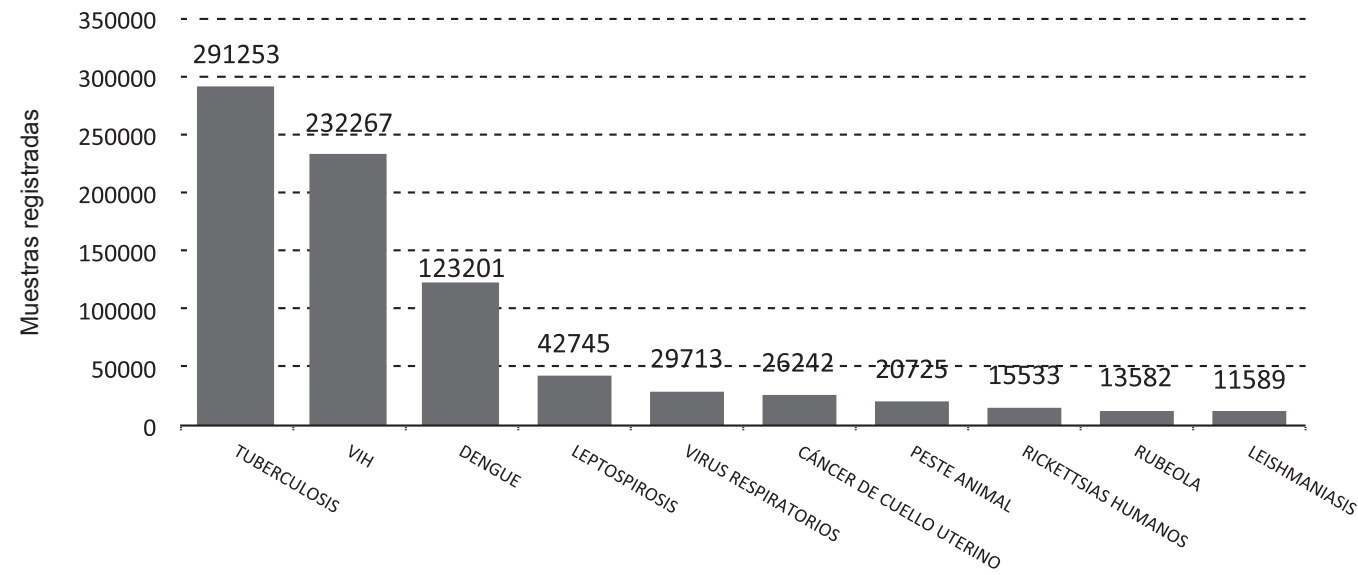

Figura 5. Volumen de muestras registradas en NETLAB de la diez enfermedades más frecuentes en el periodo 2010-2014. Fuente: Sistema de Información NETLAB

Desde el año 2010 hasta el año 2014 se ha observado un incremento progresivo de la demanda, con un crecimiento de $39,25 \%$ desde el inicio de la década que se explica por el proceso de descentralización o transferencia tecnológica de las pruebas de laboratorio del INS a los laboratorios regionales, los cuales empezaron a registrar principalmente pruebas para el diagnóstico de tuberculosis y dengue, sin embargo, algunos laboratorios regionales, como en el caso de San Martín, ha decidido que todas las pruebas que realice su laboratorio se reporten a través de NETLAB, esta debe ser una práctica replicable en toda la red de laboratorios en salud pública.

La Figura 5, presenta la proporción de muestras para diagnóstico por enfermedad, verificándose que hasta el $70,7 \%$ de la demanda de diagnóstico corresponde a tres enfermedades: tuberculosis, dengue, y VIH Sida. En tuberculosis y VIH Sida, se realizan pruebas de monitoreo clínico de la enfermedad (CD4, CD8, carga viral, y de detección de la resistencia a los antirretrovirales para VIH Sida y pruebas de sensibilidad a drogas antituberculosas. Así mismo, NETLAB ha mostrado una gran versatilidad para adaptarse a las necesidades de incorporar nuevas pruebas, como ha ocurrido, por ejemplo, en la pandemia de Influenza H1N1 del año 2009, la incorporación de pruebas rápidas como GRIESS y MODS o pruebas moleculares (genotype $^{\circledR}$ ) para diagnóstico y cultivo de Mycobacterium tuberculosis, o más recientemente, pruebas para la detección de infección por los virus de chikungunya y virus del Ébola.

Por último, es importante destacar dos experiencias actuales. La primera, es el uso del aplicativo NETLAB como soporte de los Programas de Evaluación Externa del Desempeño (PEED) en los bancos de sangre y en el diagnóstico de algunas enfermedades. Los PEED consisten en evaluaciones interlaboratorios que utilizan NETLAB para informar a tiempo real los resultados de las mismas. En el PEED para el diagnóstico de malaria, que se realiza bajo el auspicio de la Organización Panamericana de la Salud (OPS), participan los laboratorios nacionales de malaria de América del Sur y de América Central y el Caribe, los cuales utilizan el NETLAB de INS Perú, para el registro de sus resultados.

La segunda experiencia está relacionada con la implementación del módulo de citología cérvico uterina, el cual forma parte del Plan Esperanza, que es un programa para la atención integral del cáncer. EI NETLAB se utiliza en la Red de Servicios de Salud del MINSA y de las direcciones regionales de salud a nivel nacional, para el registro de datos de pacientes, muestras y resultados de las pruebas de apoyo al diagnóstico de cáncer de cuello uterino ${ }^{(13)}$, esto permite realizar un seguimiento nominal de las mujeres que participan en el programa, experiencia que se espera sea extendida al tamizaje de cáncer de mama.

Pero, el funcionamiento de NETLAB como sistema de información no está exento de limitaciones que pueden poner en riesgo lo ganado en el posicionamiento y liderazgo del INS en la red nacional de laboratorios de salud pública. El desempeño de los sistemas de información en salud dependen de tres conjuntos de determinantes: técnicos, organizacionales y del comportamiento. Los determinantes técnicos están referidos a la normalización de los procesos de registro, procesamiento y divulgación de información y a las TIC; los determinantes del comportamiento se refieren a las motivaciones que tienen los usuarios para comprometerse con el proceso de producir información de calidad y de utilizar efectivamente la información en la toma de decisiones; y los determinantes organizacionales que corresponden a los aspectos de 
planificación, financiamiento, ejecución, evaluación y supervisión del sistema de información ${ }^{(14)}$.

En lo referido a los determinantes técnicos, es necesario actualizar las normas que rigen su funcionamiento y normalizar el proceso de creación de nuevas pruebas utilizando el Current Procedural Terminology (CPT $\left.{ }^{\circledR}\right)$. En lo referido a los determinantes organizacionales, se requiere de un equipo mínimo de administración que garantice la gestión de los procesos de registro adecuado de las muestras en concordancia con las normas, la asignación de usuarios o la creación de pruebas, así como el financiamiento para la evaluación y monitoreo permanente de su funcionamiento y en lo referido a los determinantes del comportamiento, es necesario potenciar el uso de la información en las decisiones, generando una cultura de la información.

\section{PERSPECTIVAS}

En el contexto actual, el INS con NETLAB enfrenta nuevos retos. La creciente proporción de personal sanitario que utiliza Internet y telefonía móvil, así como los esfuerzos gubernamentales que se vienen realizando para dotar de Internet, a través de la Red Dorsal de Fibra Óptica, a más de 10000 establecimientos públicos, principalmente centros y puestos de salud rurales de la sierra y selva del Perú, constituyen una oportunidad importante para fortalecer el proceso de descentralización del registro de solicitudes pruebas de laboratorio y la consulta directa de los resultados a través de NETLAB, desde los establecimientos de salud, asimismo, empezar a desplegar proyectos de telediagnóstico en el INS.

EI MINSA ha elaborado una serie de normas destinadas a la implementación de sistemas de información en el sector salud ${ }^{(15)}$. Además, los lineamientos y medidas de reforma del sector salud plantean la construcción de un sistema de información integrado orientado al gobierno y conducción del sector salud, estableciendo el desarrollo y la implementación un Repositorio Nacional de Información en Salud (REUNIS) ${ }^{(16)}$. La construcción de REUNIS implica que el NETLAB, así como otros aplicativos del sector salud, implementen estándares para el registro de los datos, en el marco del cumplimiento a la implementación de los Identificadores Estándar de Datos en Salud (IEDS), definidos en el Decreto Supremo 024-2005-SA. Por ejemplo, es básico que todos los usuarios sean identificados con su DNI, que todos establecimientos de salud sean identificados con el código del Registro Nacional de Establecimientos de Salud (RENAES), que todas las pruebas de laboratorio sean identificadas con los códigos del Current Procedural Terminology (CPT®), etc., así como implementar el estándar Health Level Seven (HL7) para el intercambio de información clínica con los sistemas de historia clínica electrónica que progresivamente se desarrollarán en el marco de la Ley 30024, que crea el Registro Nacional de Historias Clínicas Electrónicas.

\section{CONCLUSIONES}

El desarrollo y la expansión del uso de Internet, ha convertido a los sistemas basados en web, en poderosas herramientas para los LIS y los aplicativos de e-Salud en general, destacándose una variedad de sus aplicaciones para diferentes problemas de salud ${ }^{(17-20)}$. En este escenario, el INS, con el NETLAB, ha desarrollado y mantiene una experiencia de gobierno electrónico que cumple un papel fundamental en la vigilancia de la salud pública. Su proceso de implementación partió del reconocimiento de un problema de gestión: el retraso excesivo en la comunicación de resultados de pruebas de laboratorio, y ha arribado a la propuesta de una solución integral al problema. En la revisión del flujo de trabajo para obtener un resultado de laboratorio se identificaron diferentes puntos críticos y actores involucrados y se mejoró el proceso comprometiendo a todos los actores, desde los proveedores de los insumos iniciales (los pacientes y sus muestras y solicitantes de las pruebas), los procesadores de las pruebas de laboratorio (personal del laboratorio) y productores de la información, hasta los usuarios finales de la información (trabajadores de la salud, funcionarios y pacientes) ${ }^{(9)}$.

El sistema de información NETLAB ha mejorado la gestión del servicio de procesamiento de pruebas de laboratorio en el INS. Ha permitido que los funcionarios y trabajadores del INS, así como a los usuarios externos, identifiquen rápidamente el estado en que se encuentra una muestra enviada, dentro del flujo de trabajo y las dificultades asociadas; ha favorecido la disminución de errores de registro e identificación de las muestras ${ }^{(7)}$; ha mejorado el tiempo de respuesta ante los requerimientos de pruebas de laboratorio por parte del sistema de servicios de salud, no solo por la comunicación de los resultados a tiempo real, sino también por la mejora de los procesos internos. Finalmente, ha permitido un ahorro importante en costos horas-hombre y en los costos de papel y del servicio postal

El INS, con el NETLAB, se encuentra en una posición privilegiada para enfrentar los retos que demandará al MINSA, la construcción de un sistema de Información que integre a los distintos actores sanitarios, como lo demanda la reforma del sector, para el intercambio de transacciones y para la 
gestión de las nuevas redes integradas, así como el desarrollo de experiencias de Tele salud, de cara a las posibilidades de conectividad con que contará el país.

Agradecimientos: a los señores Rodolfo Carlos Flores Diaz, David Campos Pezantes y al Ing. Javier Fernando Villacorta y a todo el equipo que contribuyó a la construcción del sistema de información de laboratorios del INS.
Contribuciones de autoría: JVH participó en la concepción y diseño del artículo JVH y GGN han participado en el análisis de la información y redacción del artículo, GGN y JSJ han participado en el análisis crítico del artículo. JVH aprobó la versión final del artículo.

Fuentes de financiamiento: autofinanciado

Conflictos de interés: los autores laboran o han laborado en el Instituto Nacional de Salud.

\section{REFERENCIAS BIBLIOGRÁFICAS}

1. Sepulveda JL, Young DS. The ideal laboratory information system. Arch Pathol Lab Med. 2013 Aug;137(8):1129-40. doi: 10.5858/ arpa.2012-0362-RA.

2. Peters M, Broughton PM, Nightingale PG. Use of information technology for auditing effective use of laboratory services. J Clin Pathol. 1991 Jul;44(7):539-42.

3. Wilkinson DS. The role of technology in the clinical laboratory of the future. Clin Lab Manage Rev. 1997 Sep-Oct; 11(5):322-30.

4. Rosa-Jiménez F, Martos Melguizo L, Montijano Cabrera AM, Zambrana García JL, Adarraga Cansino MD. Impacto de los sistemas automatizados en un modelo de consulta de alta resolución. An Med Interna. 2005; 22(11):511-4.

5. Bean NH, Martin SM, Bradford H Jr. PHLIS: an electronic system for reporting public health data from remote sites. Am J Public Health. 1992 Sep;82(9):1273-6.

6. U.S. Department of Defense. DOD Global Emerging Infections Surveillance and Response System. Annual Report Fiscal Year 2002. Silver Spring, MD: DOD-GEIS; 2003.

7. García P, Vargas J, Caballero P, Calle J, Bayer A. An e-health driven laboratory information system to support HIV treatment in Peru: E-quity for laboratory personnel, health providers and people living with HIV. BMC Med Inform Decis Mak. 2009;9(50).

8. Sebastián JL, Caballero P, Sánchez J, Grant R, Podestá LE. Un paso adelante en la lucha contra el SIDA: los primeros dos años de acceso universal al tratamiento en el Perú. Lima: MINSA; 2006.

9. García PJ, Vargas J, Caballero P, Suárez V, Fuentes-Tafur L. Sistemas de información: Internet, acceso y uso por trabajadores de salud y personas viviendo con VIH/SIDA. Rev Peru Med Exp Salud Publica. 2007;24(3):254-61.

10. Pagliari C, Sloan D, Gregor P, Sullivan F, Detmer D, Kahan JP, et al. What is eHealth (4): a scoping exercise to map the field. J Med Internet Res. 2005 mar;7(1):e9.

11. Pagliari C. Design and evaluation in eHealth: challenges and implications for an interdisciplinary field. J Med Internet Res. 2007 May 27;9(2):e15.

12. Boza Dibos B. Manual de Buenas Prácticas Gubernamentales 2008: Casos de Éxito. Directorio de la Tecnocracia. Lima: Ciudadanos al Día; 2008.

13. Vargas-Herrera J, Manrique J, Nuñez MC, Sánchez P, Miraval M, Merejildo $\mathrm{M}$, et al. Aplicación de un sistema de información electrónico al programa de tamizaje de cáncer de cuello uterino. Rev Peru Med Exp Salud Publica. 2013;30(3):522-4.

14. Aqil A, Lippeveld T, Hozumi D. PRISM framework: a paradigm shift for designing, strengthening and evaluating routine health information systems. Health Policy Plan. 2009 May;24(3):217-28. doi: 10.1093/ heapol/czp010.

15. Curioso WH. eSalud en Perú: implementación de políticas para el fortalecimiento de sistemas de información en salud. Rev Panam Salud Publica. 2014;35(5-6):437-41.
16. Perú,Ministerio de Salud.Lineamientos y medidas de reforma del sector salud, Julio 2013 [Internet]. Lima: MINSA; 2013 [citado el 17 de abril de 2015]. Disponible en: http://www.minsa. gob.pe/portada/Especiales/2013/ $\mathrm{r}$ eform a / d o c u m e n t o s / documentoreforma10102013.pdf

17. Gonzalez Morán F, Muñoz Criado I, Vanaclocha $\mathrm{H}$, grupo de trabajo del Análisis de Vigilancia Epidemiológica (AVE) de la Comunidad Valenciana. La información en tiempo real. Una herramienta necesaria en vigilancia epidemiológica. Gac Sanit. 2008;22(2):162-7.

18. Caro-Murillo AM, Moreno-Iribas C, Irisarri F, Aldaz P, Napal V, Varela Santos C, et al. Evaluación del sistema de información de Navarra sobre diagnósticos de infección por el VIH, 1985-2003. Rev Esp Salud Publica. 2007; 81(4):387-98.

19. Blaya JA, Shin SS, Yagui MJ, Yale G, Suarez C, Asencios L, et al. Assessing effects of the e-Chasqui laboratory information system on accuracy and timeliness of bacteriology results in the Peruvian tuberculosis program. AMIA Annu Symp Proc. 2007 Oct 11:873.

20. Harris S, Morgan M, Davies E. Webbased reporting of the results of the 2006 four country prevalence survey of healthcare associated infections. J Hosp Infect. 2008 Jul;69(3):258-64. doi: 10.1016/j.jhin.2008.04.005.

Correspondencia: Javier Vargas Herrera Dirección: Belgrano 171 -104 Pueblo Libre, Lima, Lima

Teléfono: 945029342

Correo electrónico:jvargash@unmsm.edu.pe 